\title{
The Effects of Complexity on the Product-Service Shift
}

\author{
Ella-Mae Molloy ${ }^{1}$, Dr. Carys Siemieniuch ${ }^{2}$, Murray Sinclair ${ }^{1}$ \\ ${ }^{1}$ Dept. Human Sciences, ${ }^{2}$ Dept. Electronic \& Electrical Engineering \\ Loughborough University, Loughborough, UK \\ E.Molloy@lboro.ac.uk
}

There are many challenges facing businesses in the modern commercial climate. One challenge is the 'product-service shift', whereby organisations go from delivering a product to the provision of through-life availability of an instantiated capability. This will have implications for any organisation in the processes and procedures it employs, especially for knowledge and information management. Whilst it is true that there is a large technical content within the area of knowledge and information management, it is still, essentially, a human and organisational issue. This is the focus of the KIM (Knowledge and Information Management - through life) Grand Challenge.

This paper will introduce the KIM project and aims. Focus will be on a particular area of research within the project, concerned with decision-making, decision support and human aspects of such systems. Ongoing studies within this research are discussed, looking at the bid stage for major aerospace projects and at decision networks within flagship construction projects.

The implications of complexity, in its organisational guises of intrinsic and induced complexity will be discussed.

There is a discussion on how the limited information horizon contributes to (often unwanted) emergent behaviour as projects progress, and how decision-making 
systems may be configured to help cope with issues of complexity and the surprises such emergent behaviour can manifest.

\section{Introduction}

\subsection{The KIM Grand Challenge}

The Engineering and Physical Sciences Research Council (EPSRC) in the UK has funded five Grand Challenges. All involve academic and industrial partners and aim to improve the performance of UK businesses (specifically in manufacturing and construction). 'Knowledge and Information Management - Through Life' (KIM) is the biggest of these Grand Challenges, being funded at approximately $£ 5$ million (US \$11 million).

KIM involves 11 academic institutions and number of industrial partners. Application and use in industry is a big focus of the output of this research. The overall aim of the project, as stated on the project website (www.kimproject.org), is:

"The identification of approaches to information and knowledge management that may be applied to the through-life support of long lived, complex engineered products."

The work is split into four work packages and each of those into sub tasks. Each sub task has a team of people working on it from different universities. Loughborough University is involved with several of the sub tasks. However, the focus of the research reported in this paper is the third sub task within the third work package 'Managing the Knowledge System Life Cycle'.

\subsection{The Scope of Task 3.3}

The task objective is to evaluate the potential of alternative methods of decision support in improving the quality of decision-making throughout the project lifecycle. The team personnel are from the University of Salford, the University of Cambridge, the University of Reading and Loughborough University.

The scope of the research is to investigate the effect of a shift from product delivery to through life service support on decision-making and decision support in teams 'through life'. As noted on the project website, timescales may be '10, 20 or 30 years and beyond, during which time the 'information and knowledge' will be stored, accessed, used and received many times over in many different situations and contexts.'

The issues which arise from consideration of such a time frame can be summarised, as follows: beyond 30 years we will be designing (and servicing) products and systems whose requirements and uses not yet known, using materials and processes not yet invented or developed, using suppliers who will be very different to now, and who may have a shorter lifetime than the system itself, and all of this will be done, including maintaining system information and knowledge, with people who are not yet born and who will not be in post for more than a decade. (This has been termed as 
the 'looking forwards problem'.) There are also things to be learned 'looking backwards', i.e. how are legacy systems dealt with that were first designed and produced many years ago (e.g. the RAF Canberra photo reconnaissance aeroplane, designed in the 1950s and still flying today).

\subsection{The Implications}

The implications of a shift to 'through-life' service are not insignificant, not simply due to an extension to the traditional supply chain to cover service/support providers, but also because of the extended timescales and responsibilities to be considered.

Many of the customer's harder decisions about the future are delegated to the supplier (who can no longer pass risks on along the chain). The supplier, following Ashby's Law [Ashby 1956], must create and maintain a much more complex organisational structure to assess the issues arising. Immediate considerations are: (a) provision of the capability and its upgrades on time; (b) maintaining the availability of the capability after delivery for its lifetime; and (c) the provision of a problem resolution capability to support (a) and (b) above, by addressing unexpected happenings affecting the capability.

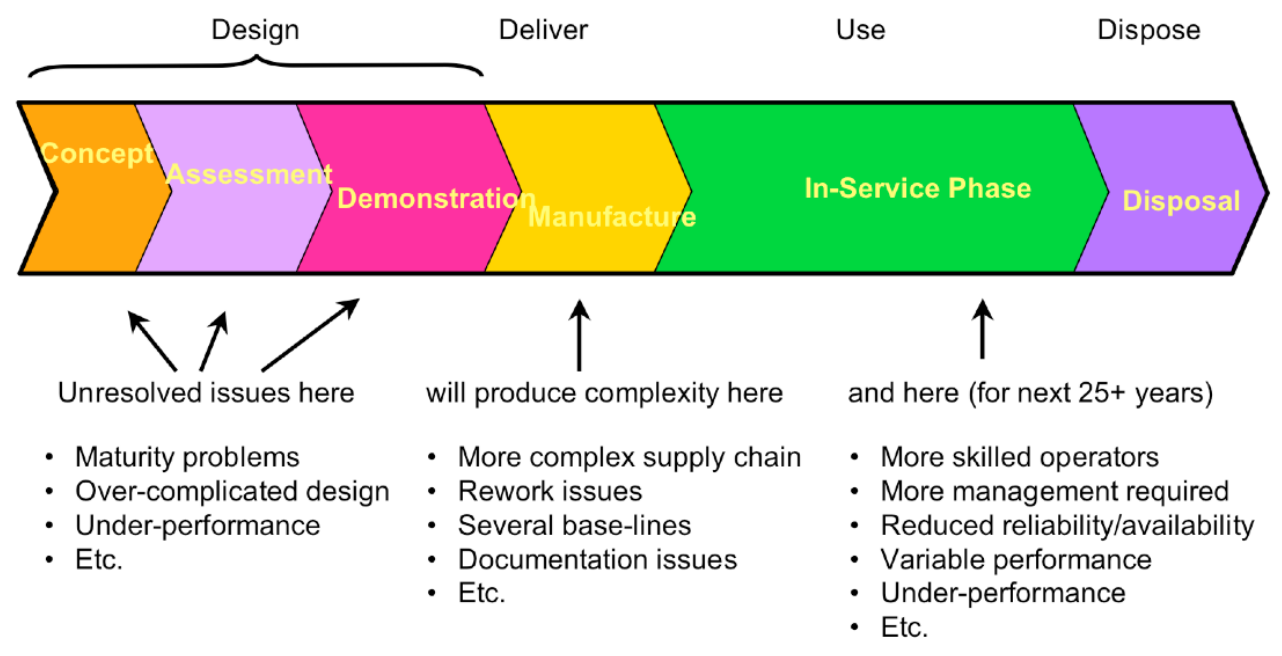

Figure 1: CADMID Lifecycle for a System Capability

The increase in effort, co-ordination and control is significant; if organisations do not rise to meet this challenge, the effects can be long lasting, as illustrated in Figure 1. This incorporates the CADMID lifecycle, well known in the UK defence industry and originally specified by the Ministry of Defence in the UK. The diagram illustrates possible knock on effects of earlier unresolved problems. 


\section{Decision Making Systems}

Decision-making is affected by a number of things. There will be external environmental and commercial pressures, but there are also internal effects and pressures. Many of these, such as established processes, organisational structures and incentivisation policies are typically determined by the company's overall strategy (for example, having an aim to work 'faster, better, cheaper' than competitors). It is important that strategy is developed and implemented in an arena of good governance, otherwise the endeavour to make good decisions will be academic and detrimental emergent behaviour will be a near certainty.

Note the use of the term Decision-Making Systems (DMS): this includes:

- Agents - software or human based, who are involved with decisions,

- Activities - the decision-making activities which enable decisions to be made,

- Infrastructure - which enables decision-making and may include computer based support,

- Knowledge and information - necessary for decision-making.

DMS will also of course be affected by time (time available in which to make the decision, time by when the decision must be made, time when the output or effect of the decision is realised, and the duration of the decision) and the style or process of decision-making.

In the view of the authors, DSS (Decision Support Systems) form part of the overall DMS. In much of the literature [e.g. Silver 1991, Finlay 1989] the term DSS or decision support is considered to extend only to computer based tools. However, this research widens that definition to include any form of support or guidance, which may be computer based, or from a human source, such as in Communities of Practice (CoPs) [Coakes and Clarke 2006, Wenger 2000].

\section{The Effects of Complexity}

"Complexity is really just reality without the simplifying assumptions that we make in order to understand it." [Allen et al. 2005]

This is a very neat encapsulation of complexity; however, for the purposes of this research, we have adopted a definition very similar to that of Rycroft and Kash [1999]:

"A system exhibits complexity if it is composed of many integrated entities of heterogeneous parts, which act in a coordinated way and whose behaviour is typically nonlinear."

Issues of complexity and emergence typically are often considered from two pools of interest. Firstly, there are those researchers concerned with the emergence of order from disorder, Conway's 'Game of Life' being a good example of this [Gardner 1970]. A different approach, attuned to an organisational perspective, is to consider the emergence of a different order (or disorder) from an established or planned order. This research is positioned in this second pool of interest, since it is likely to be of most interest to those in the service industries. 
Within this latter class of complexity, it is also possible to identify two sources of complexity. Intrinsic complexity is that which arises from what is being attempted in the process or in the problem being addressed. Secondly, there is induced complexity due to the way the organisation is structured and organised to deal with the problem.

\subsection{Intrinsic Complexity}

Intrinsic complexity arises largely from the interactions between components in a system, both intended and unintended. There are a number of potential solutions/ issues to consider which may help reduce intrinsic complexity (it should be realised that elimination of intrinsic complexity is impossible):

- Modularity in design can enable containment of complexity,

- System maturity, for example state of knowledge and quality of knowledge management,

- Architecture for core components/ system facets and rigid adherence to standards,

- Clear understanding of the problem context,

- A stable project environment, in terms of budget, timescales, client coherence, partners etc. |(has the biggest impact, but is the most difficult to achieve and unlikely to occur.

\subsection{Induced Complexity}

Induced complexity is perhaps the most prevalent and powerful reason why development and change projects do not deliver as planned.

Inducing extra complexity into the delivered capability via inappropriate project management (or conversely avoiding or reducing induced complexity via appropriate project management) is discussed widely in literature [de Meyer, Loch and Pich, 2002; Koskela and Howell, 2002; Williams, 2005].

However, as these authors indicate, the style of project management is the result of organisational culture, role assignment and knowledge of the problem area [Sinclair, 2007] but is not the only source of induced complexity; the client, the legal framework, and many other entities can create a project environment that is almost certain to trigger induced complexity.

\section{Ongoing studies and Future Work}

Initial empirical work included an in-depth literature review and case studies; the first set looked at student engineering groups, investigating the identification of key decision points and the mechanisms of decision making and decision support employed; the second set were based in the aerospace and construction sectors. 
Space does not permit a full description of these studies, but will look at one key output- a DMS Framework (see figure 2). The aim of the framework is to help orient organisations with regards to their issues, especially with regards to DMS. The increased information provided will allow the organisations to more appropriately configure their own DMS. It allows data to be captured and represented in a common format and an exemplar of the framework, is shown in figure 2 partially populated with issues, items etc. drawn from accident reports, the literature and the case studies themselves. The row headings are the component parts of a DMS, as explained earlier in the paper. The column headings are aspects of the overall system or system of

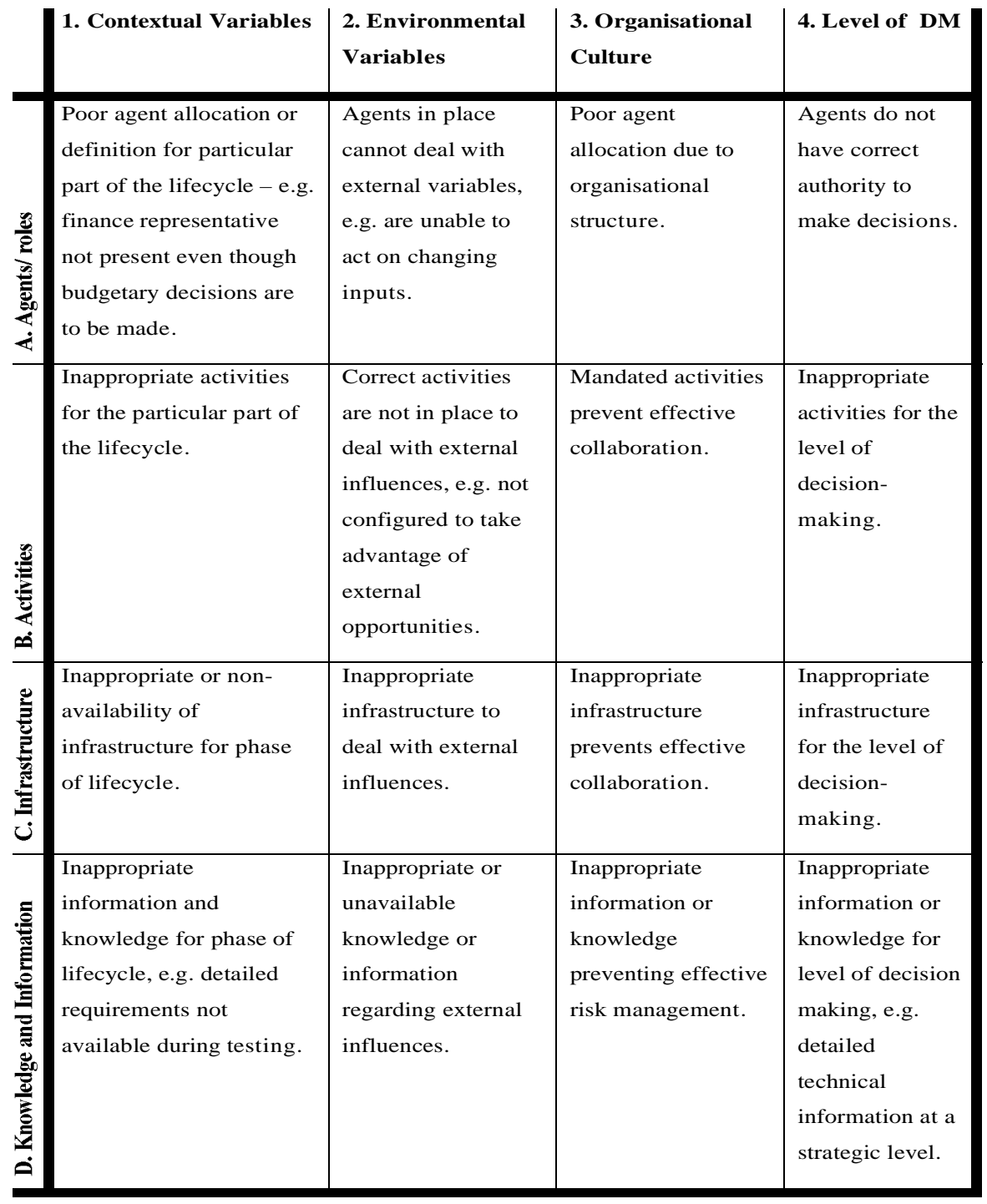

Figure 2: Example DMS framework with example contents 
systems, which could affect or be affected by the DMS. These are:

- Contextual variables - internal issues, for example, what stage are you at in the ELC (Engineering Life Cycle) and what impact does this have?

- Environmental variables- external influences, for example legislation and health and safety.

- Organisational culture, issues such as: Power distance - structure and empowerment; Risk - how much risk are the agents and the organisation willing to take? What impacts are there on the risk management system? Is it possible to take too much or too little risk?; Regimentation - what is mandated? How are things 'usually done'? What is standard practice for the organisation?; Collaboration - individual work vs. collaborative work; Level of decisionmaking (DM) - Strategic, tactical or operational.

This framework will be matured as the research continues and will be reported in more detail in the future.

\section{Conclusion}

Across all domains there is an exponential growth in the complexity of a range of long-life systems that comprise our industrialised society. We need a step-change in knowledge of how to design, integrate, operate and evolve systems a) that are not fully understood by all stakeholders b) whose behaviour may not be fully predictable and c) which function in an environment which cannot always be controlled. Complexity is an inherent feature of these systems and is characterized by:

- A (usually large) number of (usually strongly) interacting individual components of the system, and (probably) evolving interaction between the system and its environment, and;

- The requirement for the system to adapt to change in a way that does not have adverse effects on the system's usefulness, nor its ability to operate within a defined envelope of appropriate measurable parameters.

Although provider companies have in-depth expertise and knowledge about characteristics and behaviour of individual system building-block components, there is a lack of understanding of the multiple, non-linear connections and dependencies among components - and of the way that they may self-organise, or co-evolve within a constantly changing environment over its lifecycle. The response of the complex system, therefore, cannot be adequately planned, understood, nor anticipated. Understanding these issues is an incremental process. Developing tools and approaches to aid decision making to ensure the integration of hierarchies of components, sub-systems etc. and to at least allow the properties of a complex System of System (SoS) to be bounded, would be a valuable first step.

The end goal for this research is described as being able to identify key decision points and the appropriate configuration of DMS capable of delivering and servicing complex long-life systems, which must function with a high degree of uncertainty in the system operating space. The DMS must cope with:

- The containment of undesirable emergent behaviour in the delivered system, 
- Reduced timescales with controlled costs within the delivery system,

- On-going customer requirement for improved flexibility, interoperability and supportability of the system through its life cycle.

Decision support must be extended beyond rules and guidelines for each and every decision. Decision makers must be enabled with the tools and knowledge to make them aware of the likelihood of emergent behaviour of the systems and help them make resilient decisions. Any tools must be flexible enough to cope with the potential variety of events and robust enough to withstand the challenge of complexity.

\section{Bibliography}

[1] Allen, P.M., Boulton, J., Strathern, M., and Baldwin, J. (2005) The implications of complexity for business process and strategy. In K. Richardson (Ed.), Managing organisational complexity: philosophy, theory and application (pp. 397-418)

[2] Ashby, W.R. (1956) An introduction to cybernetics, London: Chapman \& Hall

[3] Coakes, E., et al. (2006). Encyclopaedia of communities of practice in information and knowledge management. London: Idea Group.

[4] de Meyer, A., Loch, C.H., and Pich, M.T. (2002) Managing project uncertainty: from variation to chaos Sloan Management Review 43(2), pp. 60-67

[5] Finlay, P. (1989). Introducing decision support systems. Oxford Manchester: NCC Blackwell.

[6] Gardner, M. (1970). The fantastic combinations of John Conway's new solitaire game "life", Scientific American, 233, October, pp. 120-123

[7] Koskela, L., and Howell, G. (2002) The theory of project management explanation to novel methods Paper presented at the Proceedings of $10^{\text {th }}$ Conference of the International Group for Lean Construction, 6-8 August 2002, Gramado, Brazil

[8] Rycroft, R.W. and Kash, D.E. (1999), The complexity challenge London: Pinter

[9] Silver, M. S. (1991). Systems that support decision makers: description and analysis. Chichester: John Wiley \& Sons.

[10] Sinclair, M.A. (2007) Ergonomics Issues in Future Systems. Keynote paper to Ergonomics Society Annual Conference, Nottingham, April 2007. Paper to be published in Ergonomics, 2007

[11] Wenger, E. (2000). Communities of Practice and Social Learning Systems. Organisation articles, 7(2), pp. 225-246.

[12] Williams, T.M. (2005) Assessing and moving on from the dominant project management discourse in the light of project overruns IEEE Transactions on Engineering Management 52(4) pp. 497-508 